\section{FOLFOX nützt vor allem Patienten mit Kolonkarzinom im Stadium III}

\begin{abstract}
FOLFOX ist ein etabliertes Therapieregime beim Kolonkarzinom. Die meisten Studiendaten zu seinem adjuvanten Einsatz sind jetzt reif und Ergebnisse gepoolter Analysen erlauben u. a. Aussagen zum Rezidivverlauf und Überleben.
\end{abstract}

$\mathrm{D}$ ie Daten von 12.223 Patienten aus 5 randomisierten, den aktuellen Studienstandards entsprechenden, Untersuchungen zur kombinierten adjuvanten Chemotherapie mit Fluorouracil (FU), Folinsäure (FS) und Oxaliplatin (zusammen FOLFOX) nach kurativer Resektion von Kolonkarzinomen im Stadium II und III sind jetzt reif.

In allen Einzelstudien (C-07, C-08, N0147, MOSAIC und XELONA) wurde der Effekt einer Zugabe von Oxaliplatin zur Therapie mit FU plus FS untersucht. Allerdings wurde nicht der Zusammenhang zwischen Patienten- und Tumorcharakteristika und dem individuellen Verlauf der Erkrankung addressiert. Die gepoolte Auswertung der ACCENT (Ad- juvant Colon Cancer End Points)-Datenbank mit den Daten der 12.223 Teilnehmern gibt nun Hinweise zum Einfluss einer Oxaliplatin-Gabe und tumorspezifischer Faktoren auf den Zeitverlauf von Rezidiven und dem Versterben. Für jeden Endpunkt wurde ein kontinuierliches Zeitrisiko über 6 Jahre modelliert. Durch den Vergleich der FU/FS-Therapie mit der Gabe von FOLFOX waren auch zeitabhängige Vergleiche möglich. Es zeigte sich, dass die Oxaliplatin-Addition das Rezidivrisiko deutlich reduzierte - bei Patienten im Stadium II innerhalb der ersten 14 Monate nach Therapiebeginn und bei Patienten im Stadium III innerhalb der ersten 4 Jahre. Bei Patienten im Stadium III verminderte Oxaliplatin zu- sätzlich das Risiko für Tod in den Jahren 2-6 nach Behandlungsbeginn. Dabei bestanden keine Unterschiede hinsichtlich des Zeitpunkts von Ergebnissen zwischen den Behandlungsgruppen. Bei Stadium-II-Patienten ließ sich das Mortalitätsrisiko in den ersten 6 Jahren durch Oxaliplatin nicht weiter reduzieren. Das Rezidiv-Maximum lag in beiden Behandlungsgruppen bei 14 Monaten. Das Risiko für Rezidiv und Tod korrelierte mit der Tumor- und Nodallast.

Fazit: Die Analyse der ACCENT-Datenbank belegt den Nutzen einer Oxaliplatin-Addition zu FU/FS für Kolonkarzinompatienten im Stadium III. Unabhängig vom adjuvanten Regime sollten die Patienten - auch im Stadium II - vor allem innerhalb der ersten 3 Jahre sorgfältig überwacht werden. Barbara Kreutzkamp

Shah MA et al. Impact of patient factors on recurrence risk and time dependency of oxaliplatin benefit in patients with colon cancer: analysis from modern-era adjuvant studies in the Adjuvant Colon Cancer End Points (ACCENT) database. J Clin Oncol. 2016;34(8):843-53.

\title{
FOLFOXIRI versus FOLFOXIRI plus Bevacizumab beim mCRC
}

\author{
Die Erstlinientherapie beim mCRC \\ wird komplexer und die Überlebens- \\ zeiten besser. In einem indirekten \\ statistischen Vergleich zweier \\ Studien fand sich ein möglicher \\ Vorteil durch eine Zugabe von \\ Bevacizumab zu FOLFOXIRI.
}

_ ür Patienten mit metastasiertem Kolorektalkarzinom (mCRC) stehen Chemotherapie-Doubletten mit Fluoropyrimidin plus Irinotecan oder Oxaliplatin, evtl. kombiniert mit einer zielgerichteten Substanz zur Verfügung. Für Patienten in gutem Allgemeinzustand kommt auch die Dreierkombinationen FOLFOXIRI infrage - mit einer möglichen weiteren Verbesserung durch Bevacizumab. Ein direkter Vergleich von FOLFOXIRI versus FOLFOXIRI plus Bevacizumab im Erstliniensetting bei mCRC-Patienten fehlt bisher. Italienische Onkologen entwickelten deshalb ein Propensity-score-adjustiertes Modell, in dem sie die Ergebnisse zweier von der GONO (Gruppo Oncologico del Nord Ovest) verantworteten Studien zusammenführten: Aus einer Phase-IIIVergleichsstudie wurden die Therapieergebnisse von mit FOLFOXIRI behandelten Patienten $(n=122)$ herangezogen, aus der TRIBE-Studie die Daten von Patienten, die FOLFOXIRI plus Bevacizumab erhalten hatten $(\mathrm{n}=252)$. Nicht ganz vermeiden ließ sich bei diesem Prozedere ein Ungleichgewicht der Patientencharakteristika in den beiden Therapiegruppen, das aber durch die Modellierung ausgeglichen werden sollte.

Das mediane progressionsfreie Überleben (PFS) von Patienten unter FOLFOXIRI plus Bevacizumab betrug 12,3 Monate im Vergleich zu 10,0 Monaten in der FOLFOXIRI-Gruppe (Propensity-score-adjustierte Hazard Ratio [HR] 0,74, 95\%-Konfidenzintervall [95\%-KI] 0,59-0,94; p = 0,013), das Ge- samtüberleben (OS) 29,8 vs. 23,6 Monate (Propensity-score-adjustierte HR 0,72, $95 \%$-KI 0,56-0,93; p = 0,014). Die Unterschiede ließen sich jeweils auch in multivariaten Modellen verifizieren. Keine Differenzen zwischen beiden Gruppen bestanden beim Ansprechen sowie der medianen Tiefe des Ansprechens.

Fazit: Bei Patienten mit mCRC erzielte die Kombination aus FOLFOXIRI plus Bevacizumab in der Neoadjuvanz signifikant bessere Ergebnisse bei PFS und OS als FOLFOXIRI ohne Bevacizumab. Die Ergebnisse des indirekten Vergleichs rechtfertigen nach Ansicht der Forscher die Zugabe von Bevacizumab zu FOLFOXIRI.

Barbara Kreutzkamp

Cremolini $C$ et al. FOLFOXIRI or FOLFOXIRI plus bevacizumab as first-line treatment of metastatic colorectal cancer: a propensity score-adjusted analysis from two randomized clinical trials. Ann Oncol. 2016 Feb 9. [Epub ahead of print]. 